\title{
Hermitian Morita Theory: a Matrix Approach
}

\author{
DAVID W. LEWIS AND THOMAS UNGER
}

ABSTRACT. In this note an explicit matrix description of hermitian Morita theory is presented.

\section{INTRODUCTION}

Let $K$ be a field of characteristic different from two and let $A$ be a central simple $K$-algebra equipped with an involution $*$. By a wellknown theorem of Wedderburn, $A$ is of the form $M_{n}(D)$, a full matrix algebra over a division $K$-algebra $D$. Furthermore, there exists an involution - on $D$ of the same kind as $*$ such that $*$ and-have the same restriction to $K$. Then $*$ is the adjoint involution $\operatorname{ad}_{h_{0}}$ of some nonsingular $\varepsilon_{0}$-hermitian form $h_{0}$ over $(D,-)$,

$$
h_{0}: D^{n} \times D^{n} \longrightarrow D \text {, }
$$

with $\varepsilon_{0}= \pm 1$. Thus

$$
X^{*}=\operatorname{ad}_{h_{0}}(X)=S \bar{X}^{t} S^{-1}, \quad \forall X \in M_{n}(D),
$$

where $S \in G L_{n}(D)$ is the matrix of $h_{0}$, so that $\bar{S}^{t}=\varepsilon_{0} S$.

Let $\operatorname{Gr}_{\varepsilon}(A, *)$ and $W_{\varepsilon}(A, *)$ denote the Grothendieck group and Witt group of $\varepsilon$-hermitian forms over $(A, *)$, respectively. Hermitian Morita theory furnishes us with isomorphisms

$$
\operatorname{Gr}_{\varepsilon}(A, *) \cong \operatorname{Gr}_{\varepsilon_{0} \varepsilon}(D,-) \text { and } W_{\varepsilon}(A, *) \cong W_{\varepsilon_{0} \varepsilon}(D,-) .
$$

These isomorphisms are the result of the following equivalences of categories

$$
\left\{\begin{array}{l}
\varepsilon \text {-hermitian } \\
\text { forms over } \\
\left(M_{n}(D), *\right)
\end{array}\right\} \stackrel{\text { scaling }}{\longleftrightarrow}\left\{\begin{array}{l}
\varepsilon_{0} \varepsilon \text {-hermitian } \\
\text { forms over } \\
\left(M_{n}(D),-{ }^{t}\right)
\end{array}\right\} \stackrel{\text { Morita }}{\longleftrightarrow \text { equivalence }}\left\{\begin{array}{l}
\varepsilon_{0} \varepsilon \text {-hermitian } \\
\text { forms over } \\
(D,-)
\end{array}\right\}
$$


(all forms are assumed to be nonsingular) which respect isometries, orthogonal sums and hyperbolic forms.

In this note we describe these correspondences explicitly. In particular we give a matrix description of Morita equivalence which does not seem to be generally known. Other explicit descriptions can be found in $[3,4,5]$. The subject is often treated in a more abstract manner, such as in [1] and [2, Chap. I, §9].

\section{SCALING}

Let $M$ be a right $M_{n}(D)$-module and let $h: M \times M \longrightarrow M_{n}(D)$ be an $\varepsilon$-hermitian form with respect to $*$, i.e.

$$
h(y, x)=\varepsilon h(x, y)^{*}=\varepsilon S \overline{h(x, y)}^{t} S^{-1} .
$$

Proposition 2.1. The form

$$
S^{-1} h: M \times M \longrightarrow M_{n}(D),(x, y) \longmapsto S^{-1} h(x, y)
$$

is $\varepsilon_{0} \varepsilon$-hermitian over $\left(M_{n}(D),-{ }^{t}\right)$.

Proof. Sesquilinearity of $S^{-1} h$ with respect to $-{ }^{t}$ follows easily from sesquilinearity of $h$ with respect to $*$ :

$$
\begin{aligned}
\left(S^{-1} h\right)(x \alpha, y) & =S^{-1} h(x \alpha, y)=S^{-1} \alpha^{*} h(x, y) \\
& =S^{-1} S \bar{\alpha}^{t} S^{-1} h(x, y)=\bar{\alpha}^{t} S^{-1} h(x, y)
\end{aligned}
$$

for any $\alpha \in M_{n}(D)$ and any $x, y \in M$.

Furthermore, using the fact that $\bar{S}^{t}=\varepsilon_{0} S$, we get

$$
\begin{aligned}
\left(S^{-1} h\right)(y, x) & =S^{-1} h(y, x) \\
& =S^{-1} \varepsilon S \overline{h(x, y)}^{t} S^{-1} \\
& =\varepsilon \overline{h(x, y)}^{t} S^{-1} \\
& =\varepsilon \varepsilon_{0} \overline{h(x, y)}^{t}{\overline{\left(S^{-1}\right)}}^{t} \\
& =\varepsilon \varepsilon_{0}{\overline{\left(S^{-1} h\right)(x, y)}}^{t}
\end{aligned}
$$

for any $x, y \in M$.

Remark 2.2. By the first part of the proof, scaling of a sesquilinear form $h$ (rather than an $\varepsilon$-hermitian form $h$ ) with respect to $*$ results in a sesquilinear form $S^{-1} h$ with respect to $-^{t}$. 
Remark 2.3. The matrix $S$ is not determined uniquely, but only up to scalar multiplication by $\lambda \in K$, since $\lambda S$ and $S$ give the same involution $\operatorname{ad}_{h_{0}}$. Hence the scaling correspondence is not canonical.

\section{Morita Equivalence}

Every module over $M_{n}(D) \cong \operatorname{End}_{D}\left(D^{n}\right)$ is a direct sum of simple modules, namely copies of $D^{n}$. Let $\left(D^{n}\right)^{k}$ be such a module. We identify $\left(D^{n}\right)^{k}$ with $D^{k \times n}$, the $k \times n$-matrices over $D$. We view each row of a $k \times n$-matrix over $D$ as an element of $D^{n}$. Note that $M_{n}(D)$ acts on $D^{k \times n}$ on the right.

Now let

$$
h: D^{k \times n} \times D^{k \times n} \longrightarrow M_{n}(D)
$$

be an $\varepsilon$-hermitian form over $\left(M_{n}(D),-^{t}\right)$.

Proposition 3.1. There exists an $\varepsilon$-hermitian $k \times k$-matrix $B \in$ $M_{k}(D)$ such that

$$
h(x, y)=\bar{x}^{t} B y, \forall x, y \in D^{k \times n} .
$$

Proof. Let $B=\left(b_{i j}\right)$. We will determine the entries $b_{i j}$. Let $e_{i j} \in$ $D^{k \times n}, e_{i j}^{\prime} \in D^{n \times k}$ and $E_{i j} \in M_{n}(D)$ respectively denote the $k \times n$ matrix, the $n \times k$-matrix and the $n \times n$-matrix with 1 in the $(i, j)$-th position and zeroes everywhere else. One can easily verify that

$$
e_{i f} E_{f \ell}=e_{i \ell},
$$

where $1 \leq i \leq k$ and $1 \leq f, \ell \leq n$. Also note that if $C \in M_{n}(D)$, then computing the product $E_{i j} C$ picks the $j$-th row of $C$ and puts it in row $i$ while making all other entries zero. Similarly, computing the product $C E_{i j}$ picks the $i$-th column of $C$ and puts it in column $j$ while making all other entries zero. The matrices $e_{i j}$ and $e_{i j}^{\prime}$ behave in a similar fashion.

The matrices $\left\{e_{i j} \mid 1 \leq i \leq k, 1 \leq j \leq n\right\}$ generate $D^{k \times n}$ as a right $M_{n}(D)$-module. Thus it suffices to compute $h\left(e_{i f}, e_{j g}\right)$ where $1 \leq i, j \leq k$ and $1 \leq f, g \leq n$. Let us first compute $h\left(e_{i i}, e_{j j}\right)$ :

$$
\begin{aligned}
h\left(e_{i i}, e_{j j}\right) & =h\left(e_{i i} E_{i i}, e_{j j} E_{j j}\right) \\
& =E_{i i} h\left(e_{i i}, e_{j j}\right) E_{j j} \\
& =m_{i j} E_{i j},
\end{aligned}
$$

where $m_{i j}$ is the $(i, j)$-th entry of $h\left(e_{i i}, e_{j j}\right) \in M_{n}(D)$. In other words, the matrix $h\left(e_{i i}, e_{j j}\right)$ has only one non-zero entry, namely $m_{i j}$ in position $(i, j)$. 
Next, let us compute $h\left(e_{i f}, e_{j g}\right)$. We will use the fact that

$$
e_{i f}=e_{i i} E_{i f}
$$

where $1 \leq i \leq k$ and $1 \leq f \leq n$, which follows from (2). We get

$$
\begin{aligned}
h\left(e_{i f}, e_{j g}\right) & =h\left(e_{i i} E_{i f}, e_{j j} E_{j g}\right) \\
& =E_{f i} h\left(e_{i i}, e_{j j}\right) E_{j g} \\
& =\left(h\left(e_{i i}, e_{j j}\right)\right)_{i j} E_{f g} \\
& =m_{i j} E_{f g} .
\end{aligned}
$$

Let $b_{i j}=m_{i j}$ where $1 \leq i, j \leq k$. We have

$$
\begin{aligned}
{\overline{e_{i f}}}^{t} B e_{j g} & =e_{f i}^{\prime} B e_{j g} \\
& =b_{i j} E_{f g} \\
& =m_{i j} E_{f g} .
\end{aligned}
$$

Therefore, $h\left(e_{i f}, e_{j g}\right)={\overline{e_{i f}}}^{t} B e_{j g}$ where $1 \leq i, j \leq k$ and $1 \leq f, g \leq$ $n$, which establishes (1).

Finally,

$$
m_{j i} E_{j i}=h\left(e_{j j}, e_{i i}\right)=\varepsilon{\overline{h\left(e_{i i}, e_{j j}\right)}}^{t}=\varepsilon \bar{m}_{i j} E_{j i}, \text { for } 1 \leq i, j \leq k,
$$

which implies $m_{j i}=\varepsilon \overline{m_{i j}}$, for $1 \leq i, j \leq k$. In other words, $\overline{m_{j i}}=$ $\varepsilon m_{i j}$, for $1 \leq i, j \leq k$, so that $\bar{B}^{t}=\varepsilon B$, which finishes the proof.

So, given an $\varepsilon$-hermitian form $h$ over $\left(M_{n}(D),-^{t}\right)$, we have obtained an $\varepsilon$-hermitian form over $(D,-)$ with matrix $B$ as in Proposition 3.1. Conversely, given an $\varepsilon$-hermitian form

$$
\varphi: D^{k} \times D^{k} \longrightarrow D
$$

represented by the matrix $B$ (i.e., $B=\left(\varphi\left(e_{i}, e_{j}\right)\right)$ for a $D$-basis $\left\{e_{i}\right\}$ of $D^{k}$ ), we define

$$
h: D^{k \times n} \times D^{k \times n} \longrightarrow M_{n}(D)
$$

by

$$
h(x, y):=\bar{x}^{t} B y, \forall x, y \in D^{k \times n},
$$

which gives an $\varepsilon$-hermitian form over $\left(M_{n}(D),-^{t}\right)$.

Remark 3.2. The correspondence $h \leftrightarrow \varphi$ already works for forms that are just sesquilinear, without assuming any hermitian symmetry. Since scaling also preserves sesquilinearity, as remarked earlier, we conclude that the category equivalences of $\S 1$ already hold for 
sesquilinear forms over $\left(M_{n}(D), *\right),\left(M_{n}(D),-^{t}\right)$ and $(D,-)$, respectively.

\section{REFERENCES}

[1] A. Fröhlich and A. M. McEvett, Forms over rings with involution, J. Algebra 12 (1969), 79-104.

[2] M.-A. Knus, Quadratic and Hermitian forms over rings, Grundlehren der Mathematischen Wissenschaften 294, Springer-Verlag, Berlin, 1991.

[3] D. W. Lewis, Forms over real algebras and the multisignature of a manifold, Advances in Math. 23 (1977), no. 3, 272-284.

[4] C. Riehm, Effective equivalence of orthogonal representations of finite groups, J. Algebra 196 (1997), no. 1, 196-210.

[5] C. Riehm, Orthogonal, symplectic and unitary representations of finite groups, Trans. Amer. Math. Soc. 353 (2001), no. 12, 4687-4727.

David Lewis and Thomas Unger,

School of Mathematical Sciences,

University College Dublin,

Belfield, Dublin 4, Ireland,

david.lewis@ucd.ie,thomas.unger@ucd.ie

Received on 19 December 2008. 